\title{
Study on the mechanism of rhynchophylline in improving obese hypertension through JAK2 / STAT3 pathway
}

\author{
Li HUANG ${ }^{1 *}$ (D), Mingjuan ZHANG ${ }^{1}$, Jinrong XIONG ${ }^{2}$
}

\begin{abstract}
Objective: The paper studied the effects of Rhynchophylline on the leptin-mediated JAK2/STAT3 pathway in hypothalamus. Using the leptin / leptin receptor and leptin-mediated JAK2/STAT3 pathway as an entry point, the research evaluated the effect of the hypotensive drug, and clarified the preliminary mechanism, expecting to provide new therapeutic ideas for essentially improving obese hypertension, balancing the body's neuro-endocrine function, and preventing the occurrence of cardiovascular diseases. Methods: A model of obese hypertensive rats was established, and the serum lipids, Lep, AngII, NPY and NO contents in each group of rats were measured. Immunohistochemical staining was used to observe the distribution of leptin receptor (LepR) and angiotensin II type 1 receptor (AT1R) in hypothalamus and aorta of rats. Western Blot method was employed to detect JAK2, STAT3, SOCS3, LepR and AT1R protein expressions in the hypothalamus of rats. After the third ventricle injection of AG490, Western blot was applied to detect the expressions of various proteins. Results: Rhynchophylline can significantly reduce AngII, Lep, and NPY levels in serum circulation. Immunohistochemistry showed that the hypothalamus and aorta AT1R distribution was significantly reduced, the hypothalamic LepR distribution increased, and the aortic LepR distribution decreased. The expressions of JAK2, LepR, and STAT3 proteins in the hypothalamus increased significantly, and the expressions of SOCS3 and AT1R proteins significantly decreased. After injection of AG490 in the third ventricle of Rhynchophylline rats, the expressions of JAK2 and STAT3 in the hypothalamus decreased significantly, while the SOCS3expression increased remarkably. Conclusion: Rhynchophylline can reduce blood lipid, as well as serum AngII, Lep, and NPY levels, increase serum NO levels in obese hypertension rats. Rhynchophylline can effectively inhibit the expression of SOCS3 by activating the JAK2/STAT3 pathway in the hypothalamus. Therefore, it is speculated that the JAK2/STAT3 signaling pathway is the main pathway through which Rhynchophylline plays a role in improving selective leptin resistance and reducing blood pressure and weight.
\end{abstract}

Keywords: obese hypertension; Rhynchophylline; hypothalamus; JAK2/STAT3 signaling pathway.

Practical Application: Rhynchophylline in improving obese hypertension.

\section{Introduction}

Obese hypertension, also known as obesity-related hypertension, refers to a disease in which obesity and hypertension coexist. As an independent risk factor for essential hypertension, obesity features a strong synergistic effect with hypertension. Data from the Framingham Cardiac Research show that $65 \%-75 \%$ of patients with essential hypertension are concurrently obese (Rahmouni, 2014; Downes Jessica, 2012). The nature of obesity is a food/energy imbalance (DeMarco et al., 2014). Leptin secreted by adipose tissue plays an indispensable role in regulating appetite and energy metabolism. After binding to specific transport proteins, leptin crosses the blood-brain barrier and binds to long leptin receptors in the ventral medial, lateral, and arcuate nucleus of the hypothalamus, transferring the energy storage of adipose tissue to the hypothalamus. The hypothalamus regulates feeding behavior, energy metabolism, water balance, and weight changes through the feeding and satiety center, and also regulates changes in blood pressure through the hypothalamus-adrenocortical axis (HPA), sympathetic-adrenal medulla system (SAM), and hypothalamus-pituitary-thyroid Axis (HPT) (Simonds et al., 2014; Fellmann et al., 2013).

The effect of Rhynchophylline is to eliminate phlegm and calm wind, invigorate the spleen and remove dampness. Modern research has confirmed that Rhynchophylline has definite effects in reducing blood pressure, blood lipids, improving insulin resistance, and protecting vascular endothelium. The present paper established a rat model of hypertension with leptin and leptin-mediated JAK2/STAT3 pathway as targets and hypothalamus and aorta as target organs. The effect of Rhynchophylline in reducing blood pressure and weight is studied. The paper also explored the effects of Rhynchophylline intervening thalamus blood pressure regulation center, feeding/energy consumption center, and the mechanism of improving selective leptin resistance, in order to provide new ideas and targets for the treatment of obese hypertension. 


\section{Methods}

\subsection{Model establishment of obese hypertension rats}

After one week of adaptive feeding, the rats were randomly divided into a model group and a control group. The model group rats were fed with high-fat food, while the control group rats were fed with common feed, and with free water. The rats were fed for 25 weeks under constant temperature and humidity. The weight and blood pressure which exceeded the average of the control group by $25 \%$ were used as the criterion for the successful establishment of the "obese hypertension rat model" [3], and rats that met the criteria were selected for the model group.

The grouping of Experiment 1: Obese hypertension rats were randomly divided into four groups, 10 rats in each group: the Rhynchophylline high-dose group, in which the rats were administered intragastrically with $20 \mathrm{mg} / \mathrm{kg}$ Rhynchophylline for 12 weeks; the middle dose group, in which the rats were intragastrically administered with $10 \mathrm{mg} / \mathrm{kg}$ Rhynchophylline for 12 weeks; the low-dose group, in which the rats were administered intragastrically with $5 \mathrm{mg} / \mathrm{kg}$ Rhynchophylline for 12 weeks; the model group, in which the rats were given normal saline for 12 weeks; and the control group, Wistar rats of the same week age fed with common feed, were given saline for 12 weeks.

Experiment 2 grouping: 12 Rhynchophylline rats were randomly divided into the AG490 + $20 \mathrm{mg} / \mathrm{kg}$ Rhynchophylline group (6 rats), of which the third ventricle was injected with $5 \mu \mathrm{l}$ of $2 \mu \mathrm{mol} / \mathrm{ml}$ AG490 solution. The $20 \mathrm{mg} / \mathrm{kg}$ Rhynchophylline group (6 rats), in which the third ventricle was injected with the same amount of saline.

Twelve rats in the model group were divided into the AG490 + model group (6 rats), in which the third ventricle was injected with $5 \mu \mathrm{l}$ of $2 \mu \mathrm{mol} / \mathrm{ml} \mathrm{AG} 490$ solution. And the model group ( 6 rats): the third ventricle was injected with the same amount of normal saline.

\subsection{ELISA to detect serum AngII, Lep, NPY and NO contents in each group}

The blood of rats in each group of experiment 1 were obtained, and marked at the mouth of the tube. The blood was centrifuged at 4,000 rpm for 15 minutes. The supernatant was drawn using a sterile pipette into $1.5 \mathrm{ml}$ plastic EP tubes. Samples were added, and the liquid in the wells were discarded. Each well was washed with $350 \mu \mathrm{l}$ of washing liquid. Standing for 2 minutes, the washing liquid was abandoned, and this process was repeated for 3 times. $100 \mu \mathrm{l}$ of B working solution was added to each well. The wells were incubated for 30 minutes at $37^{\circ} \mathrm{C}$ water-bathing. The wells were washed for 5 times. $90 \mu$ of substrate solution was added to each well. The wells were incubated in a $37^{\circ} \mathrm{C}$ water-bathing to develop color. Immediately after color development, the microplate was placed in a microplate reader to measure the absorbance of each well at $450 \mathrm{~nm}$.

\subsection{Expression of LepR and AT1R in hypothalamus and aorta}

The hypothalamus and aortic tissues of rats in each group of experiment 1 were taken out from the $10 \%$ neutral formaldehyde solution. After PBS washing, the tissues were dehydrated, embedded in paraffin, and sectioned. The thickness of the sections was $4 \mu \mathrm{m}$. The tissues were placed on a polylysine-coated glass slide. The tissues were baked at $65^{\circ} \mathrm{C}$ for $30 \mathrm{~min}$, dewaxed with conventional xylene, and dehydrated with gradient alcohol. The tissues were added dropwise with $3 \% \mathrm{H}_{2} \mathrm{O}_{2}$, incubated at $37^{\circ} \mathrm{C}$ for $10 \mathrm{~min}$, and rinsed three times with PBS, 5 min each time. The sections were placed in citrate buffer, repaired in heating water-bathing, heated in microwave oven of high heat, and cooled with tap water. The tissues were incubated with $3 \%$ $\mathrm{H}_{2} \mathrm{O}_{2}$ at room temperature for $10 \mathrm{~min}$ to deactivate endogenous oxidase, then washed three times with PBS, 5 min each time.

The sections were added dropwise with blocking goat serum working fluid at room temperature. After 20 minutes, the excess fluid was removed, without washing. The primary antibody, the Rabbit-anti-rat AT1R and LepR (both 1: 200 dilution) were successively added dropwise to the hypothalamus and aortic sections, respectively. After $4^{\circ} \mathrm{C}$ overnight incubation, the tissues were washed with PBS for three times, 5 minutes each time.

The tissues were added dropwise with biotinylated goat-anti-rabbit IgG secondary antibody working solution, incubated for 60 minutes at room temperature. The tissues were washed with PBS for three times, 5 minutes each time. Oxidase-labeled streptomyces ovalbumin working solution (SA/HRP) was added dropwise, and incubated at $37{ }^{\circ} \mathrm{C}$ for $20 \mathrm{~min}$. The tissues were rinsed with PBS for three times, 5 min each time. $1 \mathrm{ml}$ of $\mathrm{ddH}_{2} \mathrm{O}$ was added to the DAB color development kit. One drop of A, B, and C reagents, respectively, was mixed and dripped on the slice. The color was developed at room temperature. The reaction time was controlled under a microscope, and the reaction was terminated with distilled water. Hematoxylin counterstaining, 1\% hydrochloric acid alcohol differentiation, dehydration, vitrification, and neutral gum sealing were all performed accordingly. Photograph was taken.

\subsection{Western-blot measuring the expressions of JAK2, STAT3, SOCS3, LepR, and AT1R proteins}

The hypothalamus tissues of rats from experiment 1 and experiment 2 were obtained. $400 \mu$ l of cell lysate was added, and then $40 \mu \mathrm{l}$ of PMSF was added. The cell culture flask was gently agitated, then placed on ice for $10 \mathrm{~min}$ to lyse the cells uniformly. The cells were repeatedly pumped with a sterile syringe. The lysed product was transferred to the EP tube. The EP tube was icebathed for 30 minutes, centrifuged at $12000 \mathrm{~g}$ for 15 minutes. Following this, the supernatant was transferred to a new EP tube. $100 \mu \mathrm{l}$ of protein loading buffer was added to each tube and boiled for $5 \mathrm{~min}$. The cells were stored at $-80{ }^{\circ} \mathrm{C}$. The above samples were obtained. 12\% SDS-PAGE electrophoresis was conducted to separate the proteins. The protein bands were transferred to the PVDF membrane by wet method. The tissues were blocked at room temperature for $1 \mathrm{~h}$, and then added with the primary antibody (concentration 1:1000). The cells were incubated at $4{ }^{\circ} \mathrm{C}$ overnight. The primary antibody was eluted. The secondary antibody (1:1000) was added and incubated for $1 \mathrm{~h}$. The secondary antibody was washed away. Color development and fixation were performed by chemiluminescence. The expression of each protein was measured. 


\subsection{Statistical methods}

All data are expressed as mean \pm standard deviation (mean $\pm \mathrm{SD}$ ). Comparisons between two groups were performed using $t$-test, while comparisons between multiple groups of data $(>2)$ were conducted using One-way ANOVA. Statistical significance was found at $\mathrm{p}<0.05$. Data were analyzed using Graphpad Prism 5.0 (Graphpad Software. San Diego, CA).

\section{Result}

\subsection{The effect of Rhynchophylline on the blood lipids of rats}

The results showed that, compared with the model group, TC and LDL-C in Rhynchophylline groups of different concentrations decreased, see Figure 1.

\subsection{The effect of Rhynchophylline on the expressions of Ang II, Lep, NPY and NO proteins in rat serum}

The results showed that, compared with the Control group, the expressions of AngII, Lep and NPY in Model rat serum were significantly increased, but NO was significantly

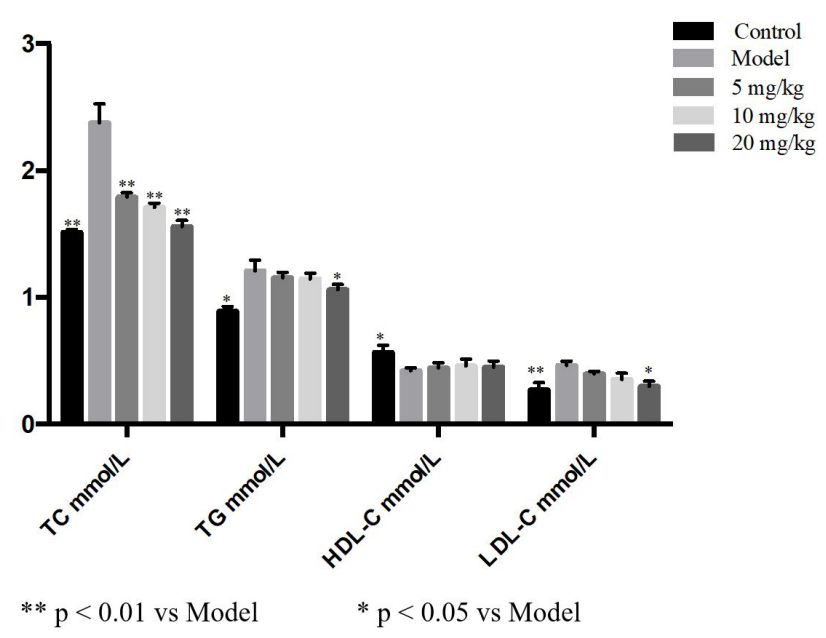

Figure 1. The effect of Rhynchophylline on blood lipids in rats. decreased. Compared with the Model group, the expressions of AngII, Lep and NPY in rats of the Rhynchophylline groups decreased significantly, while NO significantly increased, as shown in Figure 2.

\subsection{The effect of Rhynchophylline on LepR and AT1R expressions in rat hypothalamus}

Compared with the Control group, the distribution of LepR in the Model group decreased significantly. Compared with the Model group, the distribution of LepR in the drug groups increased significantly. Compared with the Control group, the distribution of AT1R in the hypothalamus in the Model group increased significantly. Compared with the Model group, the distribution of LepR and AT1R in the drug groups decreased significantly, indicating that Rhynchophylline can significantly increase the distribution of LepR and AT1R in the hypothalamus of obese hypertensive rats, as shown in Figures 3 and 4.

\subsection{Effects of Rhynchophylline on various proteins}

From Experiment 1, it can be seen that: Compared with the Control group, the expressions of hypothalamus SOCS3 and AT1R proteins in the Model group increased significantly. Compared with the Model group, Rhynchophylline can significantly reduce the expressions of hypothalamus SOCS3 and AT1R proteins. Compared with the Control group, the expressions of JAK2, STAT3, and LepR proteins in the hypothalamus of the Model group were significantly reduced. Compared with the Model group, Rhynchophylline significantly increased the expressions of JAK2, STAT3, and LepR proteins in the hypothalamus, as shown in Figure 4.

Experiment 2: After the intervention of AG490, compared with the Rhynchophylline $20 \mathrm{mg} / \mathrm{kg}$ group, the expressions of JAK2, STAT3, and LepR proteins in the AG490 + $20 \mathrm{mg} / \mathrm{kg}$ Rhynchophylline group were significantly reduced, while the expressions of SOCS3 and AT1R proteins were significantly increased. Compared with the Model group, the expressions of JAK2, STAT3, and LepR proteins in the AG490 + Model group were almost completely blocked. Compared with the AG490 + Model group, the expressions of JAK2, STAT3, and

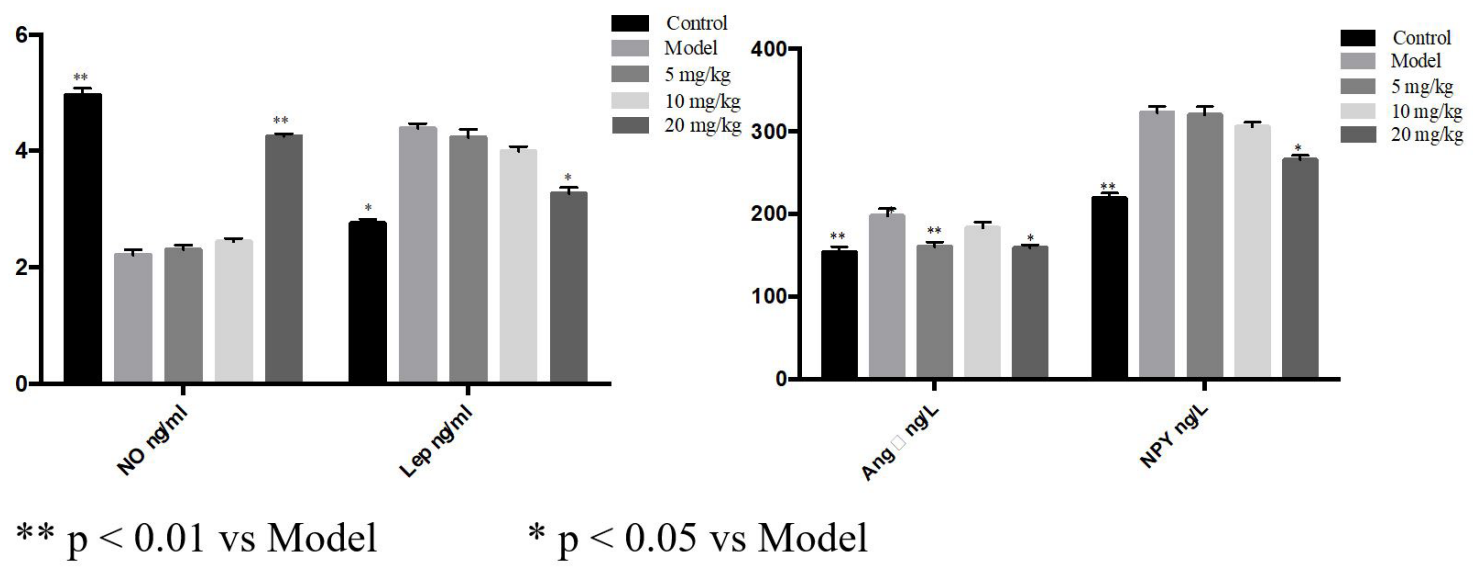

Figure 2. Effects of Rhynchophylline on the expressions of AngII, Lep, NPY and NO in rat serum. 
Control

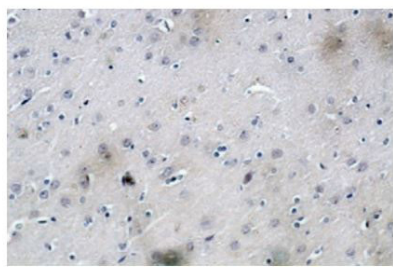

Model

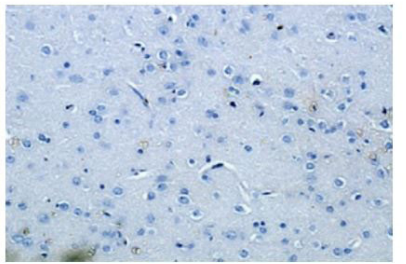

$10 \mathrm{mg} / \mathrm{kg}$
$5 \mathrm{mg} / \mathrm{kg}$

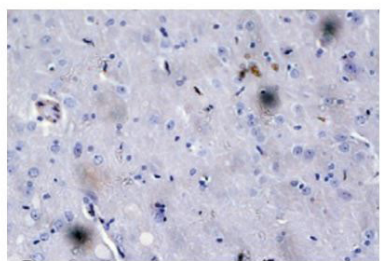

$20 \mathrm{mg} / \mathrm{kg}$
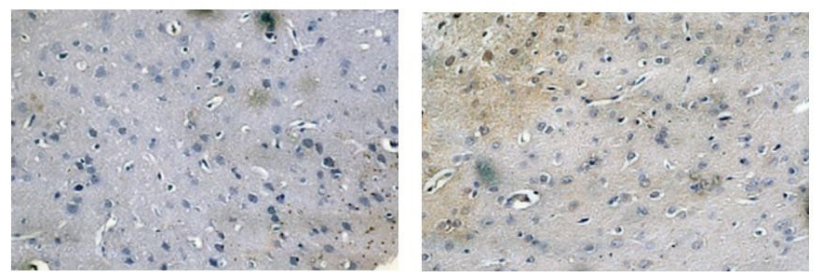

Figure 3. The effect of Rhynchophylline on the expression of LepR in the hypothalamus of rats.

\section{Control}

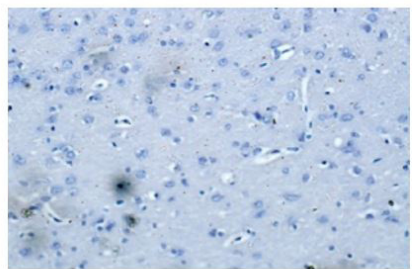

Model

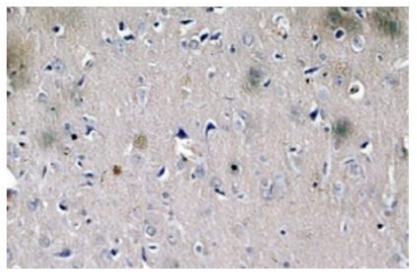

$5 \mathrm{mg} / \mathrm{kg}$

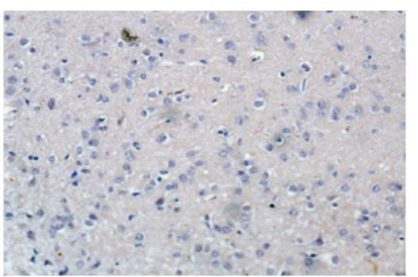

$10 \mathrm{mg} / \mathrm{kg}$

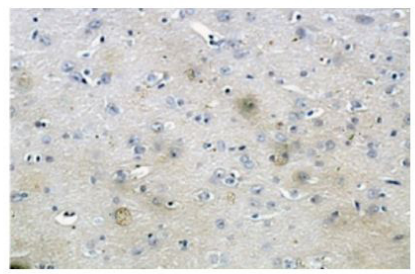

$20 \mathrm{mg} / \mathrm{kg}$

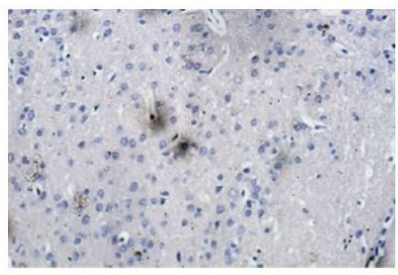

Figure 4. The effect of Rhynchophylline on the expression of AT1R in the hypothalamus of rats.

LepR proteins in the AG490 + $20 \mathrm{mg} / \mathrm{kg}$ Rhynchophylline group were still higher, while the expressions of STAT3, AT1R proteins is slightly lower, as shown in Figure 5,6.

\section{Discussion}

Obesity hypertension has become a focus of continuous research in recent years due to its high incidence, multiple risk factors and difficult treatment (Landsberg et al., 2013). Different from pure essential hypertension, obesity and hypertension have a strong synergistic effect. For example, for every $4.5 \mathrm{Kg}$ of body weight increase, systolic blood pressure increases by $4-5 \mathrm{mmHg}$ (Flegal et al., 2012). Multiple neuro-endocrine and metabolic disorders often accompanied the onset of disease, which will promote the aggregation of multiple cardiovascular risk factors. Therefore, administration with antihypertensive drugs alone is often not ideal. Reducing weight through non-drug measures such as diet control and exercise can significantly reduce blood pressure of patients, but in clinical practice, patient compliance is low. Weight-loss drugs can quickly reduce weight in a short period of time, but due to its large side effects, it is easily rebound after stopping the drug, so it is generally not recommended as the preferred treatment.

Chinese medicine has accumulated a wealth of theoretical research foundation and a large amount of clinical research 


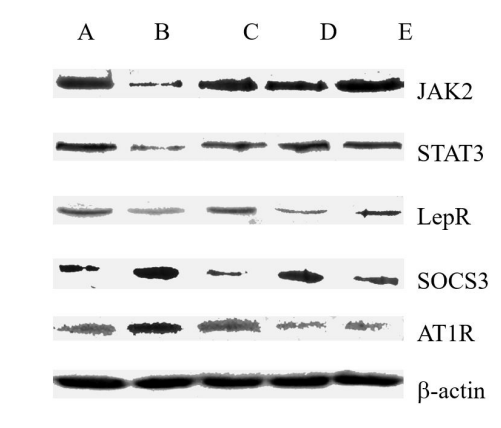

$$
\begin{aligned}
& \begin{array}{llll}
\text { A: Control } & \text { B: Model } & \text { C: } 5 \mathrm{mg} / \mathrm{kg} & \text { D: } 10 \mathrm{mg} / \mathrm{kg}
\end{array} \\
& 20 \mathrm{mg} / \mathrm{kg}
\end{aligned}
$$

Figure 5. Effect of Rhynchophylline on the expressions of various proteins in the rat hypothalamus.

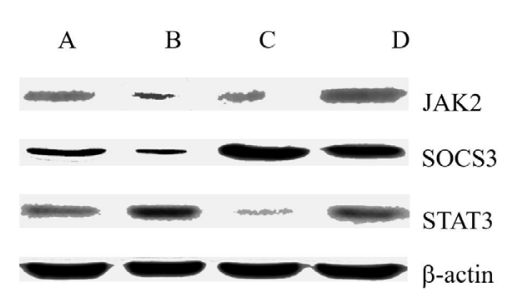

A: $A G 490+20 \mathrm{mg} / \mathrm{kg} \quad$ B: $20 \mathrm{mg} / \mathrm{kg} \quad$ C: $A G 490+$ Model $\quad$ D: Model

Figure 6. Effect of Rhynchophylline on the expressions of various proteins in rat hypothalamus after AG490 intervention.

data for the treatment of obesity and hypertension. Traditional Chinese Medicine has the characteristics of individualized treatment, overall adjustment, stable efficacy and small toxic and side effects. TCM has obvious advantages and characteristics in controlling patient weight and improving symptoms and signs such as dizziness and headache. However, the pharmacological research of many traditional Chinese medicines, especially the traditional Chinese medicine prescriptions, is not thorough, which has affected the scope of application and recognition of Chinese medicine. In this study, by evaluating the effect of Rhynchophylline on hypertension and overweight in obese hypertension rat models, and exploring the target and mechanism, the paper provided solid objective statistics for the treatment of obese hypertension by Chinese medicine, thus proposing a novel perspective for the treatment of obese hypertension.

Hyperleptinemia and selective leptin resistance are common in obese patients. The amount of leptin synthesis depends mainly on the size of the fat cells (Simonds et al., 2014), so the serum leptin in obese patients is higher than those of normal weight. The main site of leptin is in the hypothalamus. The hypothalamus is the center that regulates food intake and metabolism. Physiologically, leptin regulates the body's feeding behavior by acting on the starvation and satiety centers in its arcuate nucleus. Leptin in the circulatory system is bound to the short receptor LRa on the blood-brain barrier and then transported to the cerebrospinal fluid. Leptin binds to the long receptor LRb in the arcuate nucleus in the hypothalamus, acts on the starvation center, and inhibits NPY synthesis and release, thus reduce the appetite of the body. When leptin acts on the satiety center, it will promote the expression of POMC/CART and the synthesis of MSH. MSH combines with MC4R in the brain to suppress appetite and prevent excessive energy intake. However, in obese patients, although the level of leptin in the circulatory system increases, the increased leptin at this time cannot exert the physiological effects of suppressing appetite, increasing energy consumption, or promoting lipolysis, counteracting body fat increase, as leptin resistance occurs (Hilzendeger et al., 2012; Arias-Alvarez et al., 2010).

JAK2/STAT3 is one of the most important pathways for leptin. Inactivation or decreased JAK2/STAT3 pathway in the center system is the key to leptin resistance. AG490, a synthetic lipid derivative of phenylmethylene malononitrile, has a structure similar to tyrosine, can compete with the receptor tyrosinase for binding sites, and is a JAK2 kinase inhibitor that can specifically block the JAK2/STAT3 signaling pathway [10]. SOCS3 is not only one of the effectors but also the most important negative feedback regulator in the JAK2/STAT3 signaling pathway. Due to leptin resistance in obese hypertensive rats, the JAK2/STAT3 signaling pathway is inactivated or decreased in their hypothalamus (Hirooka et al., 2013; Saito et al., 2021).

\section{Conclusion}

In summary, Rhynchophylline can activate the JAK2/STAT3 signaling pathway in the hypothalamus, inhibit the AT1 receptor expression in the hypothalamus and the aorta, and inhibit the expression of LepR in the aorta. It is speculated that Rhynchophylline can improve leptin resistance by activating the JAK2/STAT3 signaling pathway in the hypothalamus, and exert the effect of weight loss and lowering blood pressure.

\section{Reference}

Rahmouni, K. (2014). Obesity-Associated hypertension: recent progress in deciphering the pathogenesis. Hypertension, 64(2), 215-221. http://dx.doi.org/10.1161/HYPERTENSIONAHA.114.00920. PMid:24821943.

Downes Jessica, M. (2012). An exploratory survey on the awareness and usage of clinical practice guidelines among clinical pharmacists. Exploratory Research in Clinical and Social Pharmacy, 2. http:// dx.doi.org/10.1016/J.RCSOP.2021.100013.

DeMarco, V. G., Aroor, A. R., \& Sowers, J. R. (2014). The pathophysiology of hypertension in patients with obesity. Nature Reviews. Endocrinology, 10(6), 364-376. http://dx.doi.org/10.1038/nrendo.2014.44. PMid:24732974.

Simonds, S. E., Pryor, J. T., Ravussin, E., Greenway, F. L., Dileone, R., Allen, A. M., Bassi, J., Elmquist, J. K., Keogh, J. M., Henning, E., Myers, M. G. Jr, Licinio, J., Brown, R. D., Enriori, P. J., O’Rahilly, S., Sternson, S. M., Grove, K. L., Spanswick, D. C., Farooqi, I. S., \& Cowley, M. A. (2014). Leptin mediates the increase in blood pressure associated with obesity. Cell, 159(6), 1404-1416. http:// dx.doi.org/10.1016/j.cell.2014.10.058. PMid:25480301.

Fellmann, L., Nascimento, A. R., Tibirica, E., \& Bousquet, P. (2013). Murine models for pharmacological studies of the metabolic syndrome. Pharmacology \& Therapeutics, 137(3), 331-340. http:// dx.doi.org/10.1016/j.pharmthera.2012.11.004. PMid:23178510. 
Landsberg, L., Aronne, J., Beilin, L. J., Burke, V., Igel, L. I., Lloyd-Jones, D., \& Sowers, J. (2013). Obesity-related hypertension: pathogenesis, cardiovascular risk, and treatment: a position paper of The Obesity Society and American Society of Hypertension. Journal of Clinical Hypertension, 15(1), 14-33. http://dx.doi.org/10.1111/jch.12049. PMid:23282121.

Flegal, K. M., Carroll, M. D., Kit, B. K., \& Ogden, C. L. (2012). Prevalence of obesity and trends in the distribution of body mass index among US adults, 199-2010. Journal of the American Medical Association, 307(5), 491-497. http://dx.doi.org/10.1001/jama.2012.39. PMid:22253363.

Hilzendeger, A. M., Morgan, D. A., Brooks, L., Dellsperger, D., Liu, X., Grobe, J. L., Rahmouni, K., Sigmund, C. D., \& Mark, A. L. (2012). A brain leptin-renin angiotensin system interaction in the regulation of sympathetic nerve activity. American Journal of Physiology. Heart and Circulatory Physiology, 303(2), 197-206. http://dx.doi.org/10.1152/ ajpheart.00974.2011. PMid:22610169.
Arias-Álvarez, M., García-García, R. M., Torres-Rovira, L., GonzálezBulnes, A., Rebollar, P. G., \& Lorenzo, P. L (2010). Influence of leptin on in vitro maturation and steroidogenic secretion of cumulusoocyte complexes through JAK2/STAT3 and MEK1/2 pathways in the rabbit model. Reproduction (Cambridge, England), 139(3), 523-532. http://dx.doi.org/10.1530/REP-09-0309. PMid:20032210.

Hirooka, Y., Kishi, T., Ito, K., \& Sunagawa, K. (2013). Potential clinical application of recently discovered brain mechanisms involved in hypertension. Hypertension, 62(6), 995-1002. http://dx.doi. org/10.1161/HYPERTENSIONAHA.113.00801. PMid:24101665.

Saito, M., Yajima, N., Yanai, R., Tsubokura, Y., Ichinose, K., Yoshimi, R., Ohno, S., \& Sada, K. E. (2021). Prevalence and treatment conditions for hypertension and dyslipidaemia complicated with systemic lupus erythematosus: A multi-centre cross-sectional study. Lupus, 30(7), 1146-1153. http://dx.doi.org/10.1177/09612033211006790. PMid:33794706. 